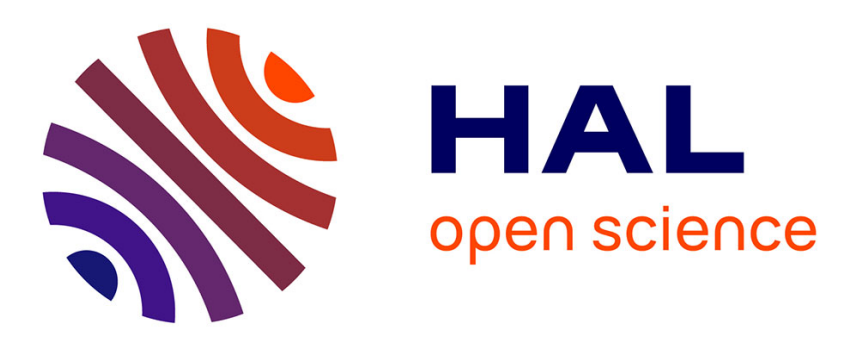

\title{
Pressure dependence of expansivity in the liquid hexane as a model for liquids
}

Ph. Pruzan

\section{To cite this version:}

$\mathrm{Ph}$. Pruzan. Pressure dependence of expansivity in the liquid hexane as a model for liquids. Journal de Physique Lettres, 1984, 45 (6), pp.273-278. 10.1051/jphyslet:01984004506027300 . jpa-00232341

\section{HAL Id: jpa-00232341 https://hal.science/jpa-00232341}

Submitted on 1 Jan 1984

HAL is a multi-disciplinary open access archive for the deposit and dissemination of scientific research documents, whether they are published or not. The documents may come from teaching and research institutions in France or abroad, or from public or private research centers.
L'archive ouverte pluridisciplinaire HAL, est destinée au dépôt et à la diffusion de documents scientifiques de niveau recherche, publiés ou non, émanant des établissements d'enseignement et de recherche français ou étrangers, des laboratoires publics ou privés. 
Classification

Physics Abstracts

$62.10-65.70$

\title{
Pressure dependence of expansivity in the liquid hexane as a model for liquids
}

\author{
Ph. Pruzan \\ Physique des Milieux Très Condensés $\left({ }^{*}\right)$, Université Pierre et Marie Curie, Tour 13, $4^{\mathrm{e}}$ étage, \\ 4, place Jussieu, 75230 Paris Cedex 05, France
}

(Reçu le 7 novembre 1983, accepté le 18 janvier 1984)

\begin{abstract}
Résumé. - A température constante, le coefficient de dilatation de l'hexane liquide obéit à une fonction en puissance de la pression jusqu'à plusieurs centaines de $\mathrm{MPa}$. On observe que le pôle de cette fonction est situé à proximité du lieu estimé de la spinodale de l'état liquide. L'examen des données montre que cette propriété s'étend à d'autres liquides. Une forme similaire peut être utilisée pour décrire la variation de la compressibilité isotherme avec la pression.
\end{abstract}

\begin{abstract}
Along isothermal paths, the expansivity of liquid hexane is shown to follow a power law up to a few hundred MPa. The pole of this function is found to be close to the expected limits of thermodynamic stability (spinodal) of the liquid state. Analysis of argon and carbon dioxide data shows that the model may be extended to other liquids. A similar equation may be used to fit the isothermal compressibility data.
\end{abstract}

In the liquid state, and roughly for pressures below $200 \mathrm{MPa}$, the expansivity $\alpha \equiv V^{-1}(\partial V / \partial T)_{\mathrm{p}}$, the isothermal compressibility $\kappa_{\mathrm{T}}=-V^{-1}(\partial V / \partial p)_{\mathrm{T}}$ and the specific heat at constant pressure $C_{\mathrm{p}} \equiv T(\partial S / \partial T)_{\mathrm{p}}$ all increase significantly as the gas phase region is approached. This increase becomes steeper within the metastable range (superheated liquid) or as the critical region is approached. An explanation of that behaviour may be provided through the spinodal concept. Below the critical temperature, metastable phases of a system can be bounded by a line of thermodynamic stability limits usually referred to as the spinodal line $[1,2]$. In the case of a onecomponent fluid, quantities such as $\alpha, \kappa_{\mathrm{T}}$ and $C_{\mathrm{p}}$ are expected to diverge as this line is approached. This may condition the behaviour of these quantities in the stable states. The van der Waals loop in the pressure volume diagram illustrates the spinodal concept of a one-component fluid. More recent models, see e.g. [3], as well as simulation methods [2] provide convincing arguments for this concept.

However for liquid systems, owing to the difficulty in investigating far into this range, there is not at the present time clear evidence for such a concept. Thus, this concept is referred to by different authors as the pseudospinodal assumption $[4,5]$. Sufficiently close to the critical region, the temperature dependence of given thermodynamic coefficients, along any isochore, is observed to follow a power law $[5,6]$. The divergence temperature locus is determined by extrapolation and

(*) ERA au CNRS No 978 . 
the treatment cannot settle questions about the spinodal [4]. The pseudospinodal assumption was successfully used to derive an equation of state (EOS) for liquid-gas systems [7].

Also of particular interest for this concept are the experimental data concerning superheated water. They show that the isobaric temperature dependences of properties such as $\alpha$ and $\kappa_{\mathrm{T}}$ may be fitted by a power law $[8,9]$. The striking feature here is the large range of validity of these laws. Despite the fact that measurements could not be performed too far into the metastable state, the divergence temperature is considered by authors as a thermodynamic stability limit [10, 12]. Generally speaking, it should be noted that the use of power laws has turned out to be very effective not only to describe phenomena close to critical regions but also states which are supposed to be bounded by a line of stability limits, such as supercooled and superheated water $[10,12]$, or the region before melting in sodium chloride [13] and ammonia [14].

Therefore, in spite of the above-mentioned difficulty, the spinodal concept is widely used. During this work, we found it convenient to adopt it. In particular, throughout this paper, the word spinodal is used to facilitate comparisons with other works. However, it should be noted that, strictly speaking, this concept must be used with care. For the time being, it must be considered rather as an empirical way of describing experimental results. It is clear that a word like pseudospinodal is better suited to the analysis of experiments concerning equilibrium states.

In order to fit the observed increases of $\alpha$ and $\kappa_{\mathrm{T}}$ mentioned above, we adopted a treatment which may be compared to the one used in the case of superheated water. The test was again to use power laws to describe the main terms of these thermodynamic coefficients. However, this treatment was applied to the isothermal pressure dependence and in a very large range concerning the stable liquid state. This test was used as an empirical guideline to fit new data on the expansivity of liquid hexane measured along isothermal paths. First attempts showed that this test was worthwhile. Along isothermal paths, which cover a surprisingly large pressure range, $\alpha$ was observed merely to follow a power law. Despite the fact that measurements were made outside the metastable state, the parameters of this power law (pole and exponent) were observed to be close to what is expected near the spinodal. Analysis of argon and carbon dioxide data in the liquid range exhibited the same features. In the same range, the isothermal compressibility of these three liquids was also found to be fairly well represented by a power law.

The expansivity of stable liquid hexane was obtained through measurements of the heat of compression for 15 isotherms lying between $240 \mathrm{~K}$ to $473 \mathrm{~K}$, up to $400 \mathrm{MPa}$ (or $700 \mathrm{MPa}$ for $300 \mathrm{~K}$ and $473 \mathrm{~K}$ ) giving a total of 295 experimental points. This method has been fully described elsewhere [15]. The results and some experimental devices will be described in another paper. The above-mentioned test concerns $a$ priori the behaviour of the thermodynamic coefficients in a rather low pressure range. Thus, we first considered only the data points of the twelve isotherms above $300 \mathrm{~K}$ and for pressures below $200 \mathrm{MPa}$. Using a non-linear least-squares method the first trials were made for each of these isotherms, the test function for $\alpha$ being given by :

$$
\alpha=A\left(p-p^{*}\right)^{-\gamma}
$$

where $p$ is the pressure and $A, p^{*}$ (the divergence pressure), $\gamma$ are parameters. The parameter $\gamma$ was found to be close to $1 / 2$. On the other hand, $A$ and $p^{*}$ were found to be accurately described by polynomials in $T$. Then, trials were made on the whole set of data up to the higher pressures of our investigation. They showed that additional terms to (1) are not required. A minimum residual sum of squares was obtained by substituting in (1) polynomials forms for $A$ and $p^{*}$, of degree 2 in temperature $T$, that is :

$$
\begin{aligned}
& A=A_{0}-A_{1} T+A_{2} T^{2} \\
& p^{*}=p_{\mathrm{c}}-a\left(T_{\mathrm{c}}-T\right)-b\left(T_{\mathrm{c}}-T\right)^{2}
\end{aligned}
$$

where the $A_{i}$ 's, $a$ and $b$ are parameters and $p_{\mathrm{c}}, T_{\mathrm{c}}$ the critical coordinates $\left(p_{\mathrm{c}}=3.03 \mathrm{MPa}\right.$, 
$T_{\mathrm{c}}=507.8 \mathrm{~K}$ ) [16]. The average relative error obtained in $\alpha$ is $5 \times 10^{-2}$ and $\gamma=1 / 2$ with a relative accuracy of $10^{-2}$. Values of the parameters in (1), (1a) and (1b) are presented in table I.

The validity of equations (1), (1a) and (1b) is illustrated for several typical isotherms in figures 1 and $1^{\prime}$ where respectively $\operatorname{Ln} \alpha$ is plotted $v s . \operatorname{Ln}\left(p-p^{*}\right)$ and $\alpha v s$. $p$. The data are well fitted, within experimental errors, even in the high pressure range. In particular, the reversal in sign of $(\partial \alpha / \partial T)_{p}$ which is very likely to be common to all liquids [17] is fairly well described. In accordance with experimental results, equations (1), (1a) and (1b) give the pressure values where $(\partial \alpha / \partial T)_{p}=0$. This locus is found to increase with $T$ from $47 \mathrm{MPa}$ at $273 \mathrm{~K}$ to $115 \mathrm{MPa}$ at $473 \mathrm{~K}$.

It is of interest to discuss the locus found for the divergence pressure $p^{*}$ and the value of the exponent $\gamma$. During the trials mentioned above several functional forms were taken for $p^{*}$. Equation (1b) provided the best fit, negligible improvements being obtained with an additional term of degree 3 in temperature. Typically, for roughly the same residual sum of squares, the observed relative variations on $p^{*}$ by changing the functional form (1b) were less than $10^{-1}$. The locus $p^{*}(T)(E q .(1 \mathrm{~b}))$ lies in the metastable range as shown in figure 2 where the vapour-pressure curve [16] and the estimates of the spinodal line for hexane are also plotted. From the experimental data, the spinodal line is estimated by extrapolation, e.g. Skripov [16] assuming that the isochores are straight lines up to the spinodal. Parmar et al. $[18,19]$ used their results on nucleation induced by an external electric field. From $423 \mathrm{~K}$ to $473 \mathrm{~K}$, the locus $p^{*}(T)$ is found to lie relatively close to the line given by Skripov. Unfortunately, the temperature in our measurements did not exceed $473 \mathrm{~K}$. From $473 \mathrm{~K}$ up to $T_{\mathrm{c}}$, we extrapolated the curve $p^{*}(T)$ which is above that of Skripov and Parmar. Below $423 \mathrm{~K}$ our results rapidly depart from the Skripov locus. For example at $373 \mathrm{~K}, p^{*}$ is $6 \mathrm{MPa}$ below this line. One may note that the Skripov EOS was built for the temperature range $393 \mathrm{~K}$ to $503 \mathrm{~K}$ and is not applicable at lower temperatures. From the surface tension data taken in the literature, a simple estimate of the spinodal is provided by the Furth model $\left[16, \mathrm{p}\right.$. 229]. It is worth noting that the $p^{*}(T)$ locus follows that estimate even very far from $T_{\mathrm{c}}$, e.g. around $273 \mathrm{~K}$.

At this point, one may question the expected behaviour of the thermodynamic coefficients around such a boundary of thermodynamic stability. Recent work, using the mean field theory [1], or an expansion of the Helmholtz potential [12], show that, as the spinodal is approached along isothermal or isobaric paths, $\alpha, \kappa_{\mathrm{T}}$ and $C_{\mathrm{p}}$ follow power laws with exponents equal to $1 / 2$. Thus, it is striking to find the locus $p^{*}(T)$ close to what is estimated for the spinodal line and a value for the exponent $\gamma$ very near to the value expected at the end-points of the metastable region. It can be suggested, for example, that $\gamma$ stays almost constant in the full metastable and stable range. Under these conditions equations (1), (1a) and (1b) may be taken as an over-all representation for the isothermal pressure dependence of the expansivity of hexane.

Table I. - Values of the parameters in (1), (1a), (1b) derived $\left(^{*}\right)$ from expansivity data for liquid hexane.

\begin{tabular}{|c|c|c|}
\hline$\gamma$ & $A_{0}\left(10^{2} \mathrm{MPa}^{1 / 2} / \mathrm{K}\right)$ & $A_{1}\left(10^{5} \mathrm{MPa}^{1 / 2} / \mathrm{K}^{2}\right)$ \\
\hline $0.5 \pm 0.005$ & $2.0779 \pm 0.007$ & $5.437 \pm 0.35$ \\
$A_{2}\left(10^{8} \mathrm{MPa}^{1 / 2} / \mathrm{K}^{3}\right)$ & $a(\mathrm{MPa} / \mathrm{K})$ & $b\left(\mathrm{MPa} / \mathrm{K}^{2}\right)$ \\
\hline$(5.404 \pm 0.47)$ & $0.02368 \pm 0.002$ & $0.0011576 \pm 3 \times 10^{-5}$ \\
\hline
\end{tabular}

(*) $A_{0}, A_{1}, A_{2}, a$ and $b$ were determined by fitting all of the $p, T, \alpha$ data using equations (1), (1a), (1b) with $\gamma=1 / 2$. 

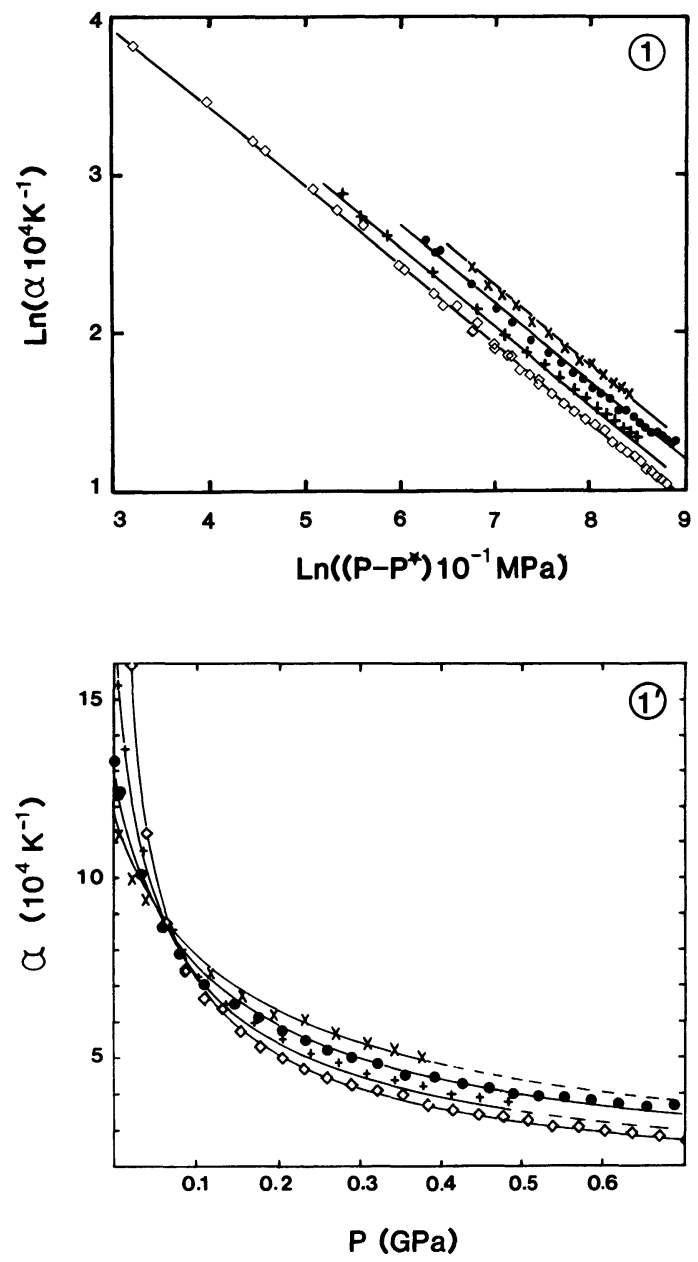

Fig. 1 and $1^{\prime}$. - Expansivity $\alpha$ of liquid hexane as a function of pressure $p .1: \operatorname{Ln} \alpha v s . \operatorname{Ln}\left(p-p^{*}\right)$. $1^{\prime}: \alpha$ vs. $p \diamond 471.5 \mathrm{~K},+374.4 \mathrm{~K}, \bullet 299.4 \mathrm{~K}, \times 251.8 \mathrm{~K}$. Solid lines, from fit of all the $p, T, \alpha$ data using equations (1), (1a), (1b).

The surprising fact that (1) holds in a very large range is not specific to liquid hexane. From the van der Waals EOS, numerical analysis shows that the exponent $\gamma$ of (1), which is $1 / 2$ at the very limit of the spinodal [20], stays close to this value for pressures up to ten times the critical pressure. More quantitative EOS, e.g. for argon [17] and carbon dioxide [21] exhibit the same features. In the almost full stable liquid state (the critical region must be excluded) the exponent $\gamma$ is close to 0.52 . Furthermore, in the case of argon, which we have studied more extensively, the $p^{*}(T)$ locus agrees accurately with the spinodal line obtained from these EOS. This feature becomes more convincing by noting that this locus is also close to the spinodal line provided by the Baidakov et al. EOS built to describe the metastable state of argon [3].

Following the test which we propose here, a fair description of the isothermal compressibility data (argon [17], carbon dioxide [21], hexane [22]) is also given, in the full liquid range, under a form similar to (1) i.e. :

$$
\kappa_{\mathrm{T}} \propto\left(p-p^{*}\right)^{-\gamma^{\prime}}
$$




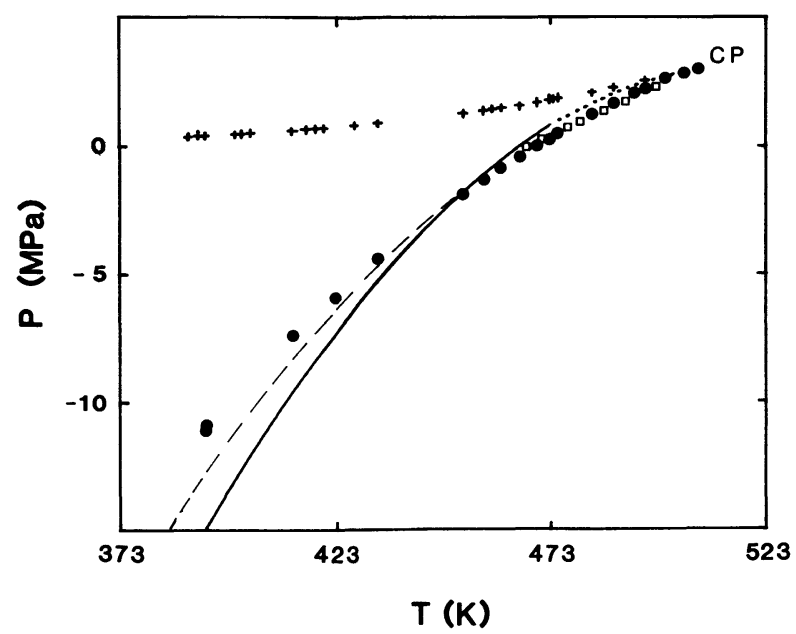

Fig. 2. - Vapour-pressure and spinodal line of hexane. + Vapour pressure curve [16]. C.P. : critical point. Solid line, $p^{*}$ locus according to this work. Dotted line, $p^{*}$ locus above $473 \mathrm{~K}$. Spinodal according to Skripov [16]. $\square$ (above $463 \mathrm{~K}$ ), spinodal according to Parmar et al. [18]. Thin broken line, spinodal according to Furth's equation [16, p. 229].

For each liquid, $\gamma^{\prime}$ is found constant within a few percent; its value lies around 0.85 . The locus $p^{*}(T)$ lies close to the region of the line $p^{*}(T)$, e.g. for argon, $p^{* \prime}$ is on average ten bars less than $p^{*}$.

The value of the exponent $\gamma^{\prime}$ is not inconsistent with the expected behaviour close to the spinodal line $[1,12]$. Numerical analysis of the different EOS suggests that very close to the spinodal line $\gamma^{\prime}$ decreases to reach the value $1 / 2$. A similar behaviour could be expected for the atmospheric temperature dependence of $\kappa_{\mathrm{T}}$ for superheated water [8 and 12]. Owing to its simple form and its range of validity, the empirical (2) can be used advantageously to fit liquid data. From the analytical view-point, equation (2) is not compatible with (1). In fact, through usual thermodynamic relationships, if equations (1), (1a), and (1b) are taken as a starting point, a functional form for $\kappa_{\mathrm{T}}-\kappa_{\mathrm{T}_{0}}$, where $T_{0}$ is a reference isotherm, may be obtained. This latter form is rather cumbersome but fulfills the conditions around the spinodal and may be used to compute $\kappa_{\mathrm{T}}$ from the $\alpha$ data.

We did not use the test mentioned above to fit the $C_{\mathrm{p}}$ data (available for argon and carbon dioxide). Information on specific heat of hexane is obtained with equations (1), (1a) and (1b) through the thermodynamic formula :

$$
\left(\partial C_{\mathrm{p}} / \partial p\right)_{\mathrm{T}}=-V T\left(\alpha^{2}+\partial \alpha / \partial T\right) .
$$

Along a given isotherm and up to a few tens of $\mathrm{MPa}, C_{\mathrm{p}}$ decreases with $p$, then it passes through a minimum and increases. Similar behaviour for other liquids has been shown from $p, V, T$ data [17]. From equations (1), (1a) and (1b) and (3), the locus of the minima of $C_{\mathrm{p}}$ may be computed for hexane. At $273 \mathrm{~K}$, the minimum is close to $100 \mathrm{MPa}$ and it moves up to $450 \mathrm{MPa}$ at $473 \mathrm{~K}$.

It has been shown that a power law fits the isothermal pressure dependence of the expansivity of hexane up to a few hundred $\mathrm{MPa}$. This functional form also applies to other liquids and provides a fair description of some properties of the liquid state. The isothermal compressibility is also observed to follow a similar law. Although these simple forms agree with some expected behaviours around the so-called spinodal, it is clear that the extrapolations in the metastable range must be interpreted with care. A closer approach to the liquid state behaviour in this range 
must be achieved by appropriate experimental investigations. Nevertheless, the observations concerning the divergence pressure locus and the exponents of these power laws suggest that a form similar to the so-called pseudospinodal assumption mentioned in the beginning, may be proposed. From a more experimental viewpoint, equations (1) and (2) may be used to fit liquid data and to build relatively simple EOS. Equations (1) and (2) are obviously not appropriate for the description of the vicinity of the critical point (roughly $1 \mathrm{~K}$ or a few bars around the critical point). In particular, the expected exponent along the critical isotherm in this zone lies in the 0.75-0.8 range. Trials to fit expansivity data of argon and carbon dioxide in this zone exhibit this trend.

\section{Acknowledgments.}

The author benefitted from stimulating discussions with Don H. Liebenberg, Dipen N. Sinha, Jacques Leblond and Henri Szwarc. Thanks are due to Philippe Arcas and Jean Michel Mariot for help in programming and Christiane Alba for measurements in hexane below $0^{\circ} \mathrm{C}$. The fruitful comments of J. M. Besson and P. Wilkinson are gratefully acknowledged.

\section{References}

[1] Compagner, A., Physica 72 (1974) 115 and references therein.

[2] Abraham, F. F., Phys. Rep. 53 (1979) 95 and references therein.

[3] Baidakov, V. G., Skripov, V. P. and Kaverin, A. M., Sov. Phys. JeTP 40 (1975) 335.

[4] Chu, B., Schoenes, F. J. and Fisher, M. E., Phys. Rev. 185 (1969) 219.

[5] Osman, J. and Sorensen, C. M., J. Chem. Phys. 73 (1980) 4142.

[6] BenedeK, G. B., in Polarisation, Matière et Rayonnement, Livre de Jubilé en l'honneur du Professeur A. Kastler (Presses Universitaires de France) 1969, p. 72.

[7] Sorensen, C. M. and Semon, M. D., Phys. Rev. A 21 (1980) 340.

[8] Hareng, M. and Leblond, J., J. Chem. Phys. 73 (1980) 622.

[9] Leblond, J. and Hareng, M., to appear in the J. Physique.

[10] SPeEdy, R. J., J. Phys. Chem. 86 (1982) 982 and references therein.

[11] Angell, C. A., Oguni, M. and Sichina, W. J., J. Phys. Chem. 86 (1982) 998 and references therein.

[12] SPeEdy, R. J., J. Phys. Chem. 86 (1982) 3002.

[13] Boyer, L. L., Phys. Rev. B 23 (1981) 3673.

[14] Pruzan, Ph., Liebenberg, D. H. and Mills, R. L., Phys. Rev. Lett. 48 (1982) 1200.

[15] Ter Minassian, L., Pruzan, Ph. and Soulard, A., J. Chem. Phys. 75 (1981) 3064.

[16] Skripov, V. P., Metastable Liquids (John Wiley and Sons, New York) 1974.

[17] Street, W. B., Physica 76 (1974) 59 and references therein.

[18] Parmar, D. S. and Jaladuddin, A. K., Phys. Lett. A 42 (1973) 497.

[19] The experimental method of Parmar and Jaladuddin was criticized by MARSTON, P. L. and APFeL, R. E., Phys. Lett. A 60 (1977) 225.

[20] We are indebted to SzWARC, H. for this remark. In fact, this result is consistent with that of [1] and [12].

[21] EOS ol Altunin and Gadetskii in carbon dioxide, International Thermodynamic Tables of the Fluid State. 3, compiled by Angus, S., Armstrong, B. and De Reuck, K. M. (Pergamon) 1976.

[22] The compressibility data of liquid hexane are available in the range $273 \mathrm{~K}$ to $353 \mathrm{~K}$. Kuss, E. and TASLIMI, M., Chem. Ing. Techn. 42 (1970) 1073;

EdulJee, H. E., Newitt, D. M. and Weale, K. E., J. Chem. Soc. 4 (1951) 3086. 